\title{
REAL HYPERSURFACES IN THE COMPLEX HYPERBOLIC QUADRIC WITH REEB INVARIANT RICCI TENSOR
}

\author{
DOO HYUN HWANG, HYUNJIN LEE, AND YOUNG JIN SUH
}

\begin{abstract}
We first give the notion of Reeb invariant Ricci tensor for real hypersurfaces $M$ in the complex quadric $Q^{m *}=S_{2, m}^{0} / S O_{2} S_{m}$, which is defined by $\mathcal{L}_{\xi}$ Ric $=0$, where Ric denotes the Ricci tensor of $M$ in $Q^{m *}$, and $\mathcal{L}_{\xi}$ the Lie derivative along the direction of the Reeb vector field $\xi=-J N$. Next we give a complete classification of real hypersurfaces in the complex hyperbolic quadric $Q^{m *}=\mathrm{SO}_{2, m}^{0} / \mathrm{SO}_{2} \mathrm{SO}_{m}$ with Reeb invariant Ricci tensor.
\end{abstract}

\section{INTRODUCTION}

Since the late 20th century there have been many studies for real hypersurfaces in the complex projective space $\mathbb{C} P^{m}$ (see [6], [8], [18, [19], [20]) and the complex hyperbolic space $\mathbb{C} H^{m}$ (see Berndt [1], Montiel and Romero [17]), which can be regarded as the class of Hermitian symmetric spaces of rank 1.

Among the class of Hermitian symmetric spaces of compact type or non-compact type with rank 2, we want to mention some examples of Riemannian symmetric spaces like $G_{2}\left(\mathbb{C}^{m+2}\right)=S U_{m+2} / S\left(U_{2} U_{m}\right)$ and $G_{2}^{*}\left(\mathbb{C}^{m+2}\right)=S U_{2, m} / S\left(U_{2} U_{m}\right)$, which are said to be complex two-plane Grassmannians and complex hyperbolic two-plane Grassmannians respectively (see 4], 21], [22, [27, [28, [29], and [30]). These are viewed as Hermitian symmetric spaces equipped with the Kähler structure $J$ and the quaternionic Kähler structure $\mathfrak{J}$.

In the class of another Hermitian symmetric space of non-compact type with rank 2, we can give the example of complex hyperbolic quadric $Q^{m *}$. It is also said to be of type (B) in Hermitian symmetric spaces. By using the method given in Kobayashi and Nomizu [13, Chapter XI, Example 10.6], the complex hyperbolic quadric $Q^{m *}=\mathrm{SO}_{2, m}^{0} / \mathrm{SO}_{2} \mathrm{SO}_{m}$ can be immersed in indefinite complex hyperbolic space $\mathbb{C H}_{1}^{m+1}$ as a space-like complex hypersurface (see Montiel and Romero [16], Romero [24], Suh [33]). The complex hyperbolic quadric $Q^{m *}$ is the non-compact

2020 Mathematics Subject Classification. Primary 53C40; Secondary 53C55.

Key words and phrases. Reeb invariant Ricci tensor, $\mathfrak{A}$-isotropic, $\mathfrak{A}$-principal, Kähler structure, complex conjugation, complex hyperbolic quadric.

The first author was supported by grant Proj. No. NRF-2021-R1C1C-2009847, the second by No. NRF-2019-R1I1A1A-01050300 and the third by No. NRF-2018-R1D1A1B-05040381 from National Research Foundation of Korea. 
Hermitian symmetric space $\mathrm{SO}_{2, m}^{0} / \mathrm{SO}_{2} \mathrm{SO}_{m}$ of rank 2 and also can be regarded as a kind of real Grassmann manifold of all oriented space-like 2-dimensional subspaces in indefinite flat Riemannian space $\mathbb{R}_{2}^{m+2}$ (see Montiel and Romero [15, 16]). Accordingly, the complex hyperbolic quadric admits both a complex conjugation structure $A$ and a Kähler structure $J$, which anti-commute with each other, that is, $A J=-J A$. Then for $m \geq 2$ the triple $\left(Q^{m *}, J, g\right)$ is a Hermitian symmetric space of compact type with rank 2 and its maximal sectional curvature is equal to -4 .

Montiel and Romero [15] proved that the complex hyperbolic quadric $Q^{m *}$ can be immersed in the indefinite complex hyperbolic space $\mathbb{C} H_{1}^{m+1}(-c), c>0$, by interchanging the Kähler metric with its opposite. Because if we change the Kähler metric of $\mathbb{C} P_{m-s}^{m+1}$ by its opposite, we have that $Q_{m-s}^{m}$ endowed with its opposite metric $g^{\prime}=-g$ is also an Einstein hypersurface of $\mathbb{C} H_{s+1}^{m+1}(-c)$. When $s=0$, we know that $\left(Q_{m}^{m}, g^{\prime}=-g\right)$ can be regarded as the complex hyperbolic quadric $Q^{m *}=\mathrm{SO}_{m, 2}^{o} / \mathrm{SO}_{2} \mathrm{SO}_{m}$, which is immersed in the indefinite complex hyperbolic quadric $\mathbb{C} H_{1}^{m+1}(-c), c>0$, as a space-like complex Einstein hypersurface.

In the paper [35] due to Suh and Hwang, we investigated the problem of commuting Ricci tensor, Ric $\phi=\phi$ Ric, for real hypersurfaces in the complex quadric $Q^{m}=S_{m+2} / S_{m} S_{2}$ and obtained the following result.

Theorem A. Let $M$ be a Hopf real hypersurface in the complex quadric $Q^{m}$, $m \geq 4$, with commuting Ricci tensor. If the shape operator commutes with the structure tensor on the distribution $\mathcal{Q}^{\perp}$, then $M$ is locally congruent to an open part of a tube around totally geodesic $\mathbb{C} P^{k}$ in $Q^{2 k}, m=2 k$ or $M$ has 3 distinct constant principal curvatures given by

$$
\begin{gathered}
\alpha=\sqrt{2(m-3)}, \gamma=0, \lambda=0, \text { and } \mu=-\frac{2}{\sqrt{2(m-3)}}, \quad \text { or } \\
\alpha=\sqrt{\frac{2}{3}(m-3)}, \gamma=0, \lambda=0, \text { and } \mu=-\frac{\sqrt{6}}{\sqrt{m-3}},
\end{gathered}
$$

with corresponding principal curvature spaces respectively

$$
T_{\alpha}=[\xi], T_{\gamma}=[A \xi, A N], \phi\left(T_{\lambda}\right)=T_{\mu}, \text { and } \operatorname{dim} T_{\lambda}=\operatorname{dim} T_{\mu}=m-2 .
$$

Remark 1.1. Besides the complex structure $J$, there is another distinguished geometric structure on the complex quadric $Q^{m}$, namely a parallel rank 2 vector bundle $\mathfrak{A}$ which contains an $S^{1}$-bundle of real structures, that is, complex conjugations $A$ on the tangent spaces of the complex quadric $Q^{m}$ (see Reckziegel [23]). This geometric structure determines a maximal $\mathfrak{A}$-invariant subbundle $\mathcal{Q}$, which is mentioned in the assumption of Theorem A of the tangent bundle $T M$ of a real hypersurface $M$ in $Q^{m}$.

Recall that a nonzero tangent vector $W \in T_{[z]} Q^{m *}$ is called singular if it is tangent to more than one maximal flat in the complex hyperbolic quadric $Q^{m *}$. There are two types of singular tangent vectors for the complex hyperbolic quadric $Q^{m *}$ :

1. If there exists a conjugation $A \in \mathfrak{A}$ such that $W \in V(A)$, then $W$ is singular. Such a singular tangent vector is called $\mathfrak{A}$-principal. 
2. If there exist a conjugation $A \in \mathfrak{A}$ and orthonormal vectors $X, Y \in V(A)$ such that $W /\|W\|=(X+J Y) / \sqrt{2}$, then $W$ is singular. Such a singular tangent vector is called $\mathfrak{A}$-isotropic,

where $V(A)=\left\{X \in T_{[z]} Q^{m *}: A X=X\right\}$ and $J V(A)=\left\{X \in T_{[z]} Q^{m *}: A X=\right.$ $-X\}$ are respectively the $(+1)$-eigenspace and (-1)-eigenspace for the involution $A$ on $T_{[z]} Q^{m *},[z] \in Q^{m *}$.

When we consider a hypersurface $M$ in the complex hyperbolic quadric $Q^{m *}$, under the assumption of some geometric properties the unit normal vector field $N$ of $M$ in $Q^{m}$ can be divided into two classes if either $N$ is $\mathfrak{A}$-isotropic or $\mathfrak{A}$-principal (see 3], 30, 33, and 34]). In the first case where $N$ is $\mathfrak{A}$-isotropic, that is, $N=(X+J Y) / \sqrt{2}$ for $X, Y \in V(A)$, Suh [33] has shown that a real hypersurface $M$ in $Q^{m *}$ with isometric Reeb flow is locally congruent to a tube over a totally geodesic $\mathbb{C} H^{k}$ in $Q^{2 k}$ or a horosphere with $\mathfrak{A}$-isotropic center at the infinity. In the second case, when the unit normal $N$ is $\mathfrak{A}$-principal, that is, $A N=N$ for a conjugation $A \in \mathfrak{A}$, we proved that a contact hypersurface $M$ in $Q^{m *}$ is locally congruent to a tube over a totally geodesic and totally real submanifold $\mathbb{R} H^{m}$ in $Q^{m *}$ (see Klein and Suh [11]).

Also motivated by Theorem A Suh and Hwang [36] gave a complete classification for real hypersurfaces in the complex hyperbolic quadric $Q^{m *}$ with commuting Ricci tensor, that is, Ric $\cdot \phi=\phi \cdot$ Ric as follows.

Theorem B (Suh and Hwang [36]). Let $M$ be a Hopf real hypersurface with commuting Ricci tensor in the complex hyperbolic quadric $Q^{m *}=\mathrm{SO}_{2, m}^{0} / \mathrm{SO}_{2} \mathrm{SO}_{m}$, $m \geq 3$. Then $M$ is locally congruent to an open part of the following manifolds:

i) a tube around totally geodesic $\mathbb{C} H^{k} \subset Q^{* 2 k}$;

ii) a horosphere whose center at infinity is $\mathfrak{A}$-isotropic singular;

iii) a hypersurface with $\mathfrak{A}$-isotropic unit normal and 3 distinct constant principal curvatures given by

$\alpha=\sqrt{\frac{2(m-3)}{2 m-5}}, \gamma=0, \lambda=\sqrt{\frac{2(m-3)}{2 m-5}}$, and $\mu=-\frac{m-2}{m-3} \sqrt{\frac{2(m-3)}{2 m-5}}$,

with corresponding principal curvature spaces respectively

$$
T_{\alpha}=[\xi], T_{\gamma}=[A \xi, A N], \phi\left(T_{\lambda}\right)=T_{\mu}, \text { and } \operatorname{dim} T_{\lambda}=\operatorname{dim} T_{\mu}=m-2 ;
$$

iv) a hypersurface with $\mathfrak{A}$-principal unit normal vector field and at most 4 distinct roots $\lambda_{1}, \lambda_{2}, \mu_{1}$, and $\mu_{2}$ satisfying the equation

$$
(2 \lambda-\alpha)^{2}+\left(\lambda^{2}-\alpha \lambda+1\right)\left\{h(2 \lambda-\alpha)-2\left(\lambda^{2}-1\right)\right\}=0,
$$

with corresponding principal curvature spaces $T_{\lambda_{1}}, T_{\lambda_{2}}, T_{\mu_{1}}$, and $T_{\mu_{2}}$ such that $V(A)=T_{\lambda_{1}} \oplus T_{\lambda_{2}} \oplus N$ and $J V(A)=T_{\mu_{1}} \oplus T_{\mu_{2}} \oplus \xi$

Remark 1.2. In Theorem $B$ ( cases i), ii), and iii) can be applied when the unit normal vector field $N$ is $\mathfrak{A}$-isotropic, and case iv) corresponds to the $\mathfrak{A}$-principal unit normal vector field $N$ in the complex hyperbolic quadric $Q^{m *}$.

Now let us consider the notion of Reeb invariant Ricci tensor for real hypersurfaces $M$ in $Q^{m *}=S O_{2, m}^{0} / S O_{2} S O_{m}$, which is given by $\mathcal{L}_{\xi}$ Ric $=0$, where Ric 
and $\mathcal{L}_{\xi}$ respectively denote the Ricci tensor of $M$ in $Q^{m *}$ and the Lie derivative along the Reeb direction $\xi=-J N$ for the Kähler structure $J$ and the unit normal vector field $N$ of $M$ in $Q^{m *}$. Then motivated by such a notion and the results mentioned above, by the help of Theorem $\mathrm{B}$, we want to give a complete classification for real hypersurfaces in the complex hyperbolic quadric $Q^{m *}$ with Reeb invariant Ricci tensor as follows.

Main Theorem. Let $M$ be a Hopf real hypersurface with Reeb invariant Ricci tensor in the complex hyperbolic quadric $Q^{m *}=S_{2, m}^{o} / S_{m} S_{2}, m \geq 3$. Then $M$ is locally congruent to an open part of the following manifolds:

i) a tube around totally geodesic $\mathbb{C} H^{k} \subset Q^{* 2 k}$;

ii) a horosphere whose center at infinity is $\mathfrak{A}$-isotropic singular;

iii) a hypersurface with $\mathfrak{A}$-isotropic unit normal and 3 distinct constant principal curvatures given by

$$
\alpha=\sqrt{\frac{2(m-3)}{2 m-5}}, \gamma=0, \lambda=\sqrt{\frac{2(m-3)}{2 m-5}}, \text { and } \mu=-\frac{m-2}{m-3} \sqrt{\frac{2(m-3)}{2 m-5}}
$$

with corresponding principal curvature spaces respectively

$$
T_{\alpha}=[\xi], T_{\gamma}=[A \xi, A N], \phi\left(T_{\lambda}\right)=T_{\mu}, \text { and } \operatorname{dim} T_{\lambda}=\operatorname{dim} T_{\mu}=m-2 ;
$$

iv) a hypersurface with $\mathfrak{A}$-principal unit normal and at most 4 distinct roots $\lambda_{1}$, $\lambda_{2}, \mu_{1}$, and $\mu_{2}$ satisfying the equation

$$
(2 \lambda-\alpha)^{2}+\left(\lambda^{2}-\alpha \lambda+1\right)\left\{h(2 \lambda-\alpha)-2\left(\lambda^{2}-1\right)\right\}=0,
$$

with corresponding principal curvature spaces $T_{\lambda_{1}}, T_{\lambda_{2}}, T_{\mu_{1}}$, and $T_{\mu_{2}}$ such that $V(A)=T_{\lambda_{1}} \oplus T_{\lambda_{2}} \oplus N$ and $J V(A)=T_{\mu_{1}} \oplus T_{\mu_{2}} \oplus \xi$.

Our paper is composed as follows. In Section 2 we present basic material about the complex quadric $Q^{m *}$, motivated by the recent work due to Klein and Suh [11]. In Section 3 , we study the geometry of the complex subbundle $\mathcal{Q}$ for real hypersurfaces in $Q^{m *}$ and some equations including Codazzi's and fundamental formulas related to the vector fields $\xi, N, A \xi$, and $A N$, where the operator $A$ denotes the complex conjugation of $M$ in the complex hyperbolic quadric $Q^{m *}$, which is explicitly constructed in Section 2 by the Lie algebraic method.

In Section 4, the first step is to derive the formula of Ricci tensor for $M$ in $Q^{m *}$ and in the next step we can show the formula of Reeb invariant Ricci tensor from the equation of Gauss for real hypersurfaces $M$ in $Q^{m *}$. Moreover, we give an important Lemma 4.2 which shows that the unit normal vector field $N$ is either $\mathfrak{A}$-isotropic or $\mathfrak{A}$-principal.

In Section 5 a complete proof of our Main Theorem with $\mathfrak{A}$-isotropic unit normal vector field will be given. In this section we prove that a real hypersurface in $Q^{m *}, m=2 k$, with invariant Ricci tensor is locally congruent to a tube over a totally geodesic $\mathbb{C} H^{k}$ in $Q^{2 k^{*}}$ or a horosphere whose center at infinity is $\mathfrak{A}$-isotropic singular.

Finally, in Section 6 we give a complete proof of our Main Theorem with $\mathfrak{A}$-principal unit normal vector field. The first part of this proof is devoted to 
studying some fundamental formulas from the Reeb invariant Ricci tensor and $\mathfrak{A}$-principal unit normal vector field. Then in the latter part we will use some trace formulas given by $\operatorname{Tr}(\phi S-S \phi)(\phi \cdot \operatorname{Ric}-\operatorname{Ric} \cdot \phi)$ and $\operatorname{Tr}(\phi \cdot \operatorname{Ric}-\operatorname{Ric} \cdot \phi)^{2}$. Then as a result we will get the formula of commuting Ricci tensor, that is, Ric $\cdot \phi=\phi \cdot$ Ric.

\section{The COMPLEX HYPERBolic QUADRIC}

In this section, let us introduce a new known result of the complex hyperbolic quadric $Q^{m *}$ different from the complex quadric $Q^{m}$ which is mentioned in [3, [1], and 32 . The $m$-dimensional complex hyperbolic quadric $Q^{m *}$ is the non-compact dual of the $m$-dimensional complex quadric $Q^{m}$, which is a kind of Hermitian symmetric space of non-compact type with rank 2 (see [5], 22], and [7]).

The complex hyperbolic quadric $Q^{m *}$ cannot be realized as a homogeneous complex hypersurface of the complex hyperbolic space $\mathbb{C} H^{m+1}$. In fact, Smyth [26. Theorem 3 (ii)] has shown that every homogeneous complex hypersurface in $\mathbb{C} H^{m+1}$ is totally geodesic. This is in marked contrast to the situation for the complex quadric $Q^{m}$, which can be realized as a homogeneous complex hypersurface of the complex projective space $\mathbb{C} P^{m+1}$ in such a way that the shape operator for any unit normal vector to $Q^{m}$ is a real structure on the corresponding tangent space of $Q^{m}$; see [10] and [23]. Another related result by Smyth [26, Theorem 1], which states that any complex hypersurface $\mathbb{C} H^{m+1}$ for which the square of the shape operator has constant eigenvalues (counted with multiplicity) is totally geodesic, also precludes the possibility of a model of $Q^{m *}$ as a complex hypersurface of $\mathbb{C} H^{m+1}$ with the analogous property for the shape operator.

Therefore we realize the complex hyperbolic quadric $Q^{m *}$ as the quotient manifold $S O_{2, m}^{0} / S_{2} S O_{m}$. As $Q^{1^{*}}$ is isomorphic to the real hyperbolic space $\mathbb{R} H^{2}=$ $S O_{1,2}^{0} / S O_{2}$, and $Q^{2^{*}}$ is isomorphic to the Hermitian product of complex hyperbolic spaces $\mathbb{C} H^{1} \times \mathbb{C} H^{1}$, we suppose $m \geq 3$ in what follows and throughout this paper. Let $G:=S_{2, m}^{0}$ be the transvection group of $Q^{m *}$ and $K:=S_{2} \mathrm{SO}_{m}$ be the isotropy group of $Q^{m *}$ at the "origin" $p_{0}:=e K \in Q^{m *}$. Then

$$
\sigma: G \rightarrow G, g \mapsto s g s^{-1}, \quad \text { with } s:=\left(\begin{array}{ccccc}
-1 & & & & \\
& -1 & & & \\
& & 1 & & \\
& & 1 & & \\
& & & \ddots & \\
& & & & 1
\end{array}\right)
$$

is an involutive Lie group automorphism of $G$ with $\operatorname{Fix}(\sigma)_{0}=K$, and therefore $Q^{m *}=G / K$ is a Riemannian symmetric space. The center of the isotropy group $K$ is isomorphic to $\mathrm{SO}_{2}$, and therefore $Q^{m *}$ is in fact a Hermitian symmetric space.

The Lie algebra $\mathfrak{g}:=\mathfrak{s o}_{2, m}$ of $G$ is given by

$$
\mathfrak{g}=\left\{X \in \mathfrak{g l}(m+2, \mathbb{R}): X^{t} \cdot s=-s \cdot X\right\}
$$

(see [12, p. 59]). In what follows we will write members of $\mathfrak{g}$ as block matrices with respect to the decomposition $\mathbb{R}^{m+2}=\mathbb{R}^{2} \oplus \mathbb{R}^{m}$, i.e. in the form

$$
X=\left(\begin{array}{ll}
X_{11} & X_{12} \\
X_{21} & X_{22}
\end{array}\right)
$$


where $X_{11}, X_{12}, X_{21}, X_{22}$ are real matrices of dimensions $2 \times 2,2 \times m, m \times 2$, and $m \times m$, respectively. Then

$$
\mathfrak{g}=\left\{\left(\begin{array}{ll}
X_{11} & X_{12} \\
X_{21} & X_{22}
\end{array}\right): X_{11}^{t}=-X_{11}, X_{12}^{t}=X_{21}, X_{22}^{t}=-X_{22}\right\} .
$$

The linearization $\sigma_{L}=\operatorname{Ad}(s): \mathfrak{g} \rightarrow \mathfrak{g}$ of the involutive Lie group automorphism $\sigma$ induces the Cartan decomposition $\mathfrak{g}=\mathfrak{k} \oplus \mathfrak{m}$, where the Lie subalgebra

$$
\begin{aligned}
\mathfrak{k} & =\operatorname{Eig}\left(\sigma_{*}, 1\right)=\left\{X \in \mathfrak{g}: s X s^{-1}=X\right\} \\
& =\left\{\left(\begin{array}{cc}
X_{11} & 0 \\
0 & X_{22}
\end{array}\right): X_{11}^{t}=-X_{11}, X_{22}^{t}=-X_{22}\right\} \\
& \cong \mathfrak{s o}_{2} \oplus \mathfrak{s o}_{m}
\end{aligned}
$$

is the Lie algebra of the isotropy group $K$, and the $2 m$-dimensional linear subspace

$$
\mathfrak{m}=\operatorname{Eig}\left(\sigma_{*},-1\right)=\left\{X \in \mathfrak{g}: s X s^{-1}=-X\right\}=\left\{\left(\begin{array}{cc}
0 & X_{12} \\
X_{21} & 0
\end{array}\right): X_{12}^{t}=X_{21}\right\}
$$

is canonically isomorphic to the tangent space $T_{p_{0}} Q^{m *}$. Under the identification $T_{p_{0}} Q^{m *} \cong \mathfrak{m}$, the Riemannian metric $g$ of $Q^{m *}$ (where the constant factor of the metric is chosen so that the formulas become as simple as possible) is given by

$$
g(X, Y)=\frac{1}{2} \operatorname{Tr}\left(Y^{t} \cdot X\right)=\operatorname{Tr}\left(Y_{12} \cdot X_{21}\right), \quad \text { for } X, Y \in \mathfrak{m} .
$$

$g$ is clearly $\operatorname{Ad}(K)$-invariant, and therefore corresponds to an $\operatorname{Ad}(G)$-invariant Riemannian metric on $Q^{m *}$. The complex structure $J$ of the Hermitian symmetric space is given by

$$
J X=\operatorname{Ad}(j) X \quad \text { for } X \in \mathfrak{m}, \quad \text { where } j:=\left(\begin{array}{ccccc}
0 & 1 & & & \\
-1 & 0 & & & \\
& & 1 & & \\
& & 1 & \\
& & & \ddots & \\
& & & & \\
& & &
\end{array}\right) \in K .
$$

Because $j$ is in the center of $K$, the orthogonal linear map $J$ is $\operatorname{Ad}(K)$-invariant, and thus defines an $\operatorname{Ad}(G)$-invariant Hermitian structure on $Q^{m *}$. By identifying the multiplication by the unit complex number $i$ with the application of the linear map $J$, the tangent spaces of $Q^{m *}$ thus become $m$-dimensional complex linear spaces, and we will adopt this point of view in what follows.

For the complex quadric, the Riemannian curvature tensor $\bar{R}$ of $Q^{m *}$ can be fully described in terms of the "fundamental geometric structures" $g, J$, and $\mathfrak{A}$. In fact, under the correspondence $T_{p_{0}} Q^{m *} \cong \mathfrak{m}$, the curvature $\bar{R}(X, Y) Z$ corresponds to - [[X,Y],Z] for $X, Y, Z \in \mathfrak{m}$; see [13, Chapter XI, Theorem 3.2(1)]. By evaluating the latter expression explicitly, one can show that one has

$$
\begin{aligned}
\bar{R}(X, Y) Z= & -g(Y, Z) X+g(X, Z) Y \\
& -g(J Y, Z) J X+g(J X, Z) J Y+2 g(J X, Y) J Z \\
& -g(A Y, Z) A X+g(A X, Z) A Y \\
& -g(J A Y, Z) J A X+g(J A X, Z) J A Y
\end{aligned}
$$

for arbitrary $A \in \mathfrak{A}_{p_{0}}$. As mentioned in the introduction, the curvature tensor of a space-like complex hypersurface $Q^{m *}$ in $\mathbb{C} H_{1}^{m+1}(-1)$ can be also obtained from the curvature tensor of $\mathbb{C} H_{1}^{m+1}(-1)$ by the equation of Gauss (see Kimura and 
Ortega [9] and Smyth 25]). Therefore the curvature of $Q^{m *}$ is the negative of that of the complex quadric $Q^{m}$; cf. [23, Theorem 1]. This confirms that the symmetric space $Q^{m *}$ which we have constructed here is indeed the non-compact dual of the complex quadric.

For any $p \in Q^{m *}$ and $A \in \mathfrak{A}_{p}$, the real structure $A$ induces a splitting

$$
T_{p} Q^{m *}=V(A) \oplus J V(A)
$$

into two orthogonal, maximal totally real subspaces of the tangent space $T_{p} Q^{m *}$. Here $V(A)$ and $J V(A)$ are the $(+1)$-eigenspace and the $(-1)$-eigenspace of $A$, respectively. For every unit vector $W \in T_{p} Q^{m *}$ there exist $t \in\left[0, \frac{\pi}{4}\right], A \in \mathfrak{A}_{p}$, and orthonormal vectors $X, Y \in V(A)$ so that

$$
W=\cos (t) X+\sin (t) J Y
$$

holds; see [23, Proposition 3]. Here $t$ is uniquely determined by $W$. The vector $W$ is singular, i.e. contained in more than one Cartan subalgebra of $\mathfrak{m}$, if and only if either $t=0$ or $t=\frac{\pi}{4}$ holds. The vectors with $t=0$ are called $\mathfrak{A}$-principal, whereas the vectors with $t=\frac{\pi}{4}$ are called $\mathfrak{A}$-isotropic. If $W$ is regular, i.e. if $0<t<\frac{\pi}{4}$ holds, then also $A$ and $X, Y$ are uniquely determined by $W$.

\section{Some General EQUATIONS}

Let $M$ be a real hypersurface in the complex hyperbolic quadric $Q^{m *}$ and denote by $(\phi, \xi, \eta, g)$ the induced almost contact metric structure. Note that $\xi=-J N$, where $N$ is a (local) unit normal vector field of $M$. The tangent bundle $T M$ of $M$ splits orthogonally into $T M=\mathcal{C} \oplus \mathbb{R} \xi$, where $\mathcal{C}=\operatorname{ker}(\eta)$ is the maximal complex subbundle of $T M$. The structure tensor field $\phi$ restricted to $\mathcal{C}$ coincides with the complex structure $J$ restricted to $\mathcal{C}$, and $\phi \xi=0$.

At each point $[z] \in M$ we define the maximal $\mathfrak{A}$-invariant subspace of $T_{[z]} M$, $[z] \in M$, as follows:

$$
\mathcal{Q}_{[z]}=\left\{X \in T_{[z]} M: A X \in T_{[z]} M \text { for all } A \in \mathfrak{A}_{[z]}\right\} .
$$

Lemma 3.1 (See [33]). For each $[z] \in M$ we have

(i) If $N_{[z]}$ is $\mathfrak{A}$-principal, then $\mathcal{Q}_{[z]}=\mathcal{C}_{[z]}$.

(ii) If $N_{[z]}$ is not $\mathfrak{A}$-principal, there exist a conjugation $A \in \mathfrak{A}$ and orthonormal vectors $X, Y \in V(A)$ such that $N_{[z]}=\cos (t) X+\sin (t) J Y$ for some $t \in$ $(0, \pi / 4]$. Then we have $\mathcal{Q}_{[z]}=\mathcal{C}_{[z]} \ominus \mathbb{C}(J X+Y)$.

We now assume that $M$ is a Hopf hypersurface. Then for the Reeb vector field $\xi$ the shape operator $S$ becomes

$$
S \xi=\alpha \xi
$$

with the smooth function $\alpha=g(S \xi, \xi)$ on $M$. When we consider a transform $J X$ of the Kähler structure $J$ on the complex hyperbolic quadric $Q^{m *}$ for any vector field $X$ on $M$ in $Q^{m *}$, we may put

$$
J X=\phi X+\eta(X) N
$$


for a unit normal $N$ to $M$. Then from the Riemannian curvature tensor of the complex hyperbolic quadric, we can induce the Codazzi equation as follows:

$$
\begin{aligned}
g\left(\left(\nabla_{X} S\right) Y-\left(\nabla_{Y} S\right) X, Z\right)= & -\eta(X) g(\phi Y, Z)+\eta(Y) g(\phi X, Z)+2 \eta(Z) g(\phi X, Y) \\
& -g(X, A N) g(A Y, Z)+g(Y, A N) g(A X, Z) \\
& -g(X, A \xi) g(J A Y, Z)+g(Y, A \xi) g(J A X, Z) .
\end{aligned}
$$

On the other hand, at each point $[z] \in M$ we can choose $A \in \mathfrak{A}_{z}$ such that

$$
N=\cos (t) Z_{1}+\sin (t) J Z_{2}
$$

for some orthonormal vectors $Z_{1}, Z_{2} \in V(A)$ and $0 \leq t \leq \frac{\pi}{4}$ (see Proposition 3 in [23]). Since $\xi=-J N$, we have

$$
\begin{aligned}
A N & =\cos (t) Z_{1}-\sin (t) J Z_{2}, \\
\xi & =\sin (t) Z_{2}-\cos (t) J Z_{1}, \\
A \xi & =\sin (t) Z_{2}+\cos (t) J Z_{1} .
\end{aligned}
$$

This implies $g(\xi, A N)=0$. From the property $J A \xi=-A J \xi=-A N$, we obtain:

Lemma 3.2 ([14] and [33]). Let $M$ be a Hopf hypersurface in the complex hyperbolic quadric $Q^{m *}$ with (local) unit normal vector field $N$. For each point in $z \in M$ we choose $A \in \mathfrak{A}_{z}$ such that $N_{z}=\cos (t) Z_{1}+\sin (t) J Z_{2}$ holds for some orthonormal vectors $Z_{1}, Z_{2} \in V(A)$ and $0 \leq t \leq \frac{\pi}{4}$. Then

$$
Y \alpha=(\xi \alpha) \eta(Y)-2 g(\xi, A N) g(Y, A \xi)+2 g(Y, A N) g(\xi, A \xi)
$$

and

$$
\begin{aligned}
0= & 2 g(S \phi S X, Y)-\alpha g((\phi S+S \phi) X, Y)+2 g(\phi X, Y) \\
& -2 g(X, A N) g(Y, A \xi)+2 g(Y, A N) g(X, A \xi) \\
& -2 g(\xi, A \xi)\{g(Y, A N) \eta(X)-g(X, A N) \eta(Y)\}
\end{aligned}
$$

holds for all vector fields $X$ and $Y$ on $M$.

Then from (2.1) and the equation of Gauss, the curvature tensor $R$ of $M$ in the complex hyperbolic quadric $Q^{m *}$ is defined so that

$$
\begin{aligned}
R(X, Y) Z= & -g(Y, Z) X+g(X, Z) Y-g(\phi Y, Z) \phi X+g(\phi X, Z) \phi Y \\
& +2 g(\phi X, Y) \phi Z-g(A Y, Z)(A X)^{T}+g(A X, Z)(A Y)^{T} \\
& -g(J A Y, Z)(J A X)^{T}+g(J A X, Z)(J A Y)^{T} \\
& +g(S Y, Z) S X-g(S X, Z) S Y,
\end{aligned}
$$

where $(A X)^{T}$ and $S$ denote the tangential component of the vector field $A X$ and the shape operator of $M$ in $Q^{m *}$, respectively. 


\section{ReEb inVARIANCE AND A Key LEMma}

Now we consider that $M$ is a real hypersurface in the complex hyperbolic quadric $Q^{m *}$. Then we may put

$$
A X=B X+\rho(X) N
$$

for any vector field $X \in T_{[z]} Q^{m *}, z \in M, \rho(X)=g(A X, N)$, where $B X$ and $\rho(X) N$ respectively denote the tangential and normal component of the vector field $A X$. Then $A \xi=B \xi+\rho(\xi) N$ and $\rho(\xi)=g(A \xi, N)=0$. It follows that

$$
\begin{aligned}
A N & =A J \xi=J A \xi=-J(B \xi+\rho(\xi) N) \\
& =-(\phi B \xi+\eta(B \xi) N) .
\end{aligned}
$$

The equation gives $g(A N, N)=-\eta(B \xi)$ and $g(A N, \xi)=0$. From this, together with the curvature tensor 3.2 for $M$ in $Q^{m *}$ in Section 3 , the Ricci tensor is given by

$$
\begin{aligned}
\operatorname{Ric}(X)= & -(2 m-1) X+3 \eta(X) \xi+g(A N, N)(A X)^{T}-g(A X, N)(A N)^{T} \\
& -g(A X, \xi) A \xi+(\operatorname{Tr} S) S X-S^{2} X
\end{aligned}
$$

where $(A X)^{T}$ denotes the tangential component to $T_{[z]} M,[z] \in M$.

On the other hand, it can be easily checked that the Ricci tensor is Reeb invariant, that is, $\mathcal{L}_{\xi}$ Ric $=0$ if and only if

$$
(\phi S-S \phi) \cdot \operatorname{Ric}=\operatorname{Ric} \cdot(\phi S-S \phi)
$$

Remark 4.1. Let $M$ be a real hypersurface over a totally geodesic $\mathbb{C} H^{k} \subset Q^{2 k^{*}}$, $m=2 k$ or a horosphere with $\mathfrak{A}$-isotropic center at the infinity. Then by a theorem due to Suh [33] the structure tensor commutes with the shape operator, that is, $S \phi=\phi S$. Moreover, the unit normal vector field $N$ becomes $\mathfrak{A}$-isotropic. This gives $\eta(B \xi)=g(A \xi, \xi)=0$. So it naturally satisfies the formula 4.2 , i.e., it is Reeb invariant.

On the other hand, from 4.2 we assert the following important lemma.

Lemma 4.2. Let $M$ be a Hopf real hypersurface in the complex hyperbolic quadric $Q^{m *}, m \geq 3$, with Reeb invariant Ricci tensor. Then the unit normal vector field $N$ becomes singular, that is, $N$ is $\mathfrak{A}$-isotropic or $\mathfrak{A}$-principal.

Proof. By putting $X=\xi$ in 4.2 we get

$$
(\phi S-S \phi) \operatorname{Ric}(\xi)=0 .
$$

Here from (4.1) the Ricci curvature along the Reeb direction $\xi$ is given by

$$
\operatorname{Ric}(\xi)=-(2 m-4) \xi+g(A N, N) A \xi-g(A \xi, \xi) A \xi+(\operatorname{Tr} S) \alpha \xi-\alpha^{2} \xi,
$$

where $g(A \xi, \xi)=g(A J N, J N)=-g(J A N, J N)=-g(A N, N)$. Substituting this one into 4.3 gives

$$
g(A N, N)(\phi S-S \phi) A \xi=0
$$


The first case gives that $g(A N, N)=g(A \xi, \xi)=\cos 2 t=0$, that is, $t=\frac{\pi}{4}$. This implies that the unit normal $N$ becomes $N=\frac{Z_{1}+J Z_{2}}{\sqrt{2}}, Z_{1}, Z_{2} \in V(A)$ from (3.1). This means that $N$ is $\mathfrak{A}$-isotropic.

The second case gives that

$$
\phi S A \xi=S \phi A \xi
$$

Similarly, we also know that

$$
\phi S(A N)^{T}=S \phi(A N)^{T},
$$

where $(A N)^{T}$ denotes the tangential component of the vector field $A N$ in $Q^{m *}$. From equations (4.4) and 4.5 we know that the shape operator $S$ commutes with the structure tensor $\phi$ on the distribution $Q^{\perp}=\operatorname{Span}\left[A \xi,(A N)^{T}\right]$.

On the other hand, by taking the inner product of (4.4) with the tangent vector field $A \xi$ we know that

$$
S \phi A \xi=\phi S A \xi=0
$$

This gives that

$$
S A \xi=\alpha \eta(A \xi) \xi
$$

By virtue of the commuting $S \phi=\phi S$ on the distribution $Q^{\perp}=\left[A \xi,(A N)^{T}\right]$, we know that $\lambda=0$ or $\lambda=\alpha$ if we put $S A N=\lambda A N$. Moreover, in papers by Suh [31, 33] we have mentioned that the distribution $Q^{\perp}$ is invariant under the shape operator $S$ if and only if $\phi S=S \phi$ on the distribution $Q^{\perp}$. Then, together with the notion of Hopf, without loss of generality we may put

$$
S \xi=\alpha \xi, \quad S A \xi=\alpha A \xi, \quad S A N=\alpha A N .
$$

From this, together with (4.6), we have for a non-vanishing Reeb function $\alpha \neq 0$

$$
A \xi=\eta(A \xi) \xi= \pm \xi
$$

When the Reeb function $\alpha$ is vanishing, by the first formula in Lemma 3.2 that is,

$$
Y \alpha=(\xi \alpha) \eta(Y)-2 g(\xi, A N) g(Y, A \xi)+2 g(Y, A N) g(\xi, A \xi),
$$

it follows that

$$
g\left(Y,(A N)^{T}\right) g(\xi, A \xi)=0 .
$$

Since in the second case we have assumed that $N$ is not $\mathfrak{A}$-isotropic, we know that $g(\xi, A \xi) \neq 0$. So it follows that $(A N)^{T}=0$. This means that

$$
A N=(A N)^{T}+g(A N, N) N=g(A N, N) N,
$$

which implies that

$$
N=A^{2} N=g(A N, N) A N=g^{2}(A N, N) N .
$$

This gives $g(A N, N)= \pm 1$, that is, we can take the unit normal $N$ such that $A N=N$. So the unit normal $N$ is $\mathfrak{A}$-principal, that is, $A N=N$. 
In order to prove our Main Theorem in the introduction, by virtue of Lemma 4.2 we are able to consider two classes of hypersurfaces in $Q^{m *}$, with the unit normal $N$ either $\mathfrak{A}$-principal or $\mathfrak{A}$-isotropic. For $M$ a real hypersurface in $Q^{m *}$ with $\mathfrak{A}$-isotropic normal vector field, in Section 5 we will give the proof in detail; in Section 6 we will give the remaining proof for the case that $M$ has a $\mathfrak{A}$-principal normal vector field.

\section{Proof of Main Theorem With $\mathfrak{A}$-isotropic unit normal Vector field}

In this section we want to prove our Main Theorem for real hypersurfaces $M$ in $Q^{m *}$ with commuting Ricci tensor when the unit normal vector field becomes $\mathfrak{A}$-isotropic.

Since we assumed that the unit normal $N$ is $\mathfrak{A}$-isotropic, by the definition in Section 3 we know that $t=\frac{\pi}{4}$. Then by the expression of the $\mathfrak{A}$-isotropic unit normal vector field, 3.1 gives $N=\frac{1}{\sqrt{2}} Z_{1}+\frac{1}{\sqrt{2}} J Z_{2}$. This implies that $g(A \xi, \xi)=0$. Then the Ricci tensor (4.1) for a real hypersurface $M$ in the complex quadric $Q^{m *}$ reduces to

$$
\operatorname{Ric}(X)=-(2 m-1) X+3 \eta(X) \xi-g(A X, N) A N-g(A X, \xi) A \xi+h S X-S^{2} X .
$$

From this, together with the fact that $A \xi=\phi A N$ and $\phi A \xi=-A N$, it follows that

$$
\begin{aligned}
\phi \cdot \operatorname{Ric}(X)= & -(2 m-1) \phi X-g(A X, N) A \xi+g(A X, \xi) A N \\
& +h \phi S X-\phi S^{2} X
\end{aligned}
$$

and

$$
\begin{aligned}
\operatorname{Ric}(\phi X)= & -(2 m-1) \phi X+g(X, A \xi) A N-g(X, A N) A \xi \\
& +h S \phi X-S^{2} \phi X,
\end{aligned}
$$

where the function $h$ denotes the trace of the shape operator $S$ of $M$ in $Q^{m *}$. Then substracting 5.2 from 5.1 gives

$$
\phi \cdot \operatorname{Ric}(X)-\operatorname{Ric}(\phi X)=h(\phi S-S \phi) X-\left(\phi S^{2}-S^{2} \phi\right) X .
$$

On the other hand, we know that the Reeb invariant Ricci tensor $\mathcal{L}_{\xi}$ Ric $=0$ is equivalent to

$$
(\phi S-S \phi) \cdot \operatorname{Ric}=\operatorname{Ric} \cdot(\phi S-S \phi) .
$$

By using the formula (5.4) and taking the trace in (5.3), we have

$$
\begin{aligned}
& \operatorname{Tr}(\phi \cdot \operatorname{Ric}-\operatorname{Ric} \cdot \phi)^{2}=\sum_{i, j} g\left(\phi \cdot \operatorname{Ric}\left(e_{i}\right)-\operatorname{Ric} \cdot \phi\left(e_{i}\right), \phi \cdot \operatorname{Ric}\left(e_{i}\right)-\operatorname{Ric} \cdot \phi\left(e_{i}\right)\right) \\
& \quad=h \operatorname{Tr}(\phi S-S \phi)(\phi \cdot \operatorname{Ric}-\operatorname{Ric} \cdot \phi)-\operatorname{Tr}\left(\phi S^{2}-S^{2} \phi\right)(\phi \cdot \operatorname{Ric}-\operatorname{Ric} \cdot \phi) \\
& \quad=-\operatorname{Tr}\left(\phi S^{2}-S^{2} \phi\right)(\phi \operatorname{Ric}-\operatorname{Ric} \phi),
\end{aligned}
$$


where in the second equality we have used (5.4) to get

$$
\begin{aligned}
\operatorname{Tr}(\phi S-S \phi)(\phi \cdot \operatorname{Ric}-\operatorname{Ric} \cdot \phi) & =\operatorname{Tr} \phi \cdot \operatorname{Ric}(\phi S-S \phi)-\operatorname{Tr}(\phi S-S \phi) \text { Ric } \cdot \phi \\
& =\operatorname{Tr} \phi(\phi S-S \phi) \cdot \operatorname{Ric}-\operatorname{Tr}(\phi S-S \phi) \text { Ric } \cdot \phi \\
& =\operatorname{Tr}(\phi S-S \phi) \operatorname{Ric} \cdot \phi-\operatorname{Tr}(\phi S-S \phi) \text { Ric } \cdot \phi \\
& =0 .
\end{aligned}
$$

On the other hand, the final term in 5.5 becomes

$$
\begin{aligned}
\operatorname{Tr}\left(\phi S^{2}-S^{2} \phi\right)(\phi \cdot \operatorname{Ric}-\operatorname{Ric} \cdot \phi) \\
\quad=\operatorname{Tr} \phi S^{2} \phi \cdot \operatorname{Ric}-\operatorname{Tr} S^{2} \phi^{2} \cdot \operatorname{Ric}-\operatorname{Tr} \phi S^{2} \operatorname{Ric} \cdot \phi+\operatorname{Tr} S^{2} \phi \cdot \operatorname{Ric} \cdot \phi \\
\quad=2 \operatorname{Tr} \phi S^{2} \phi \cdot \operatorname{Ric}-\operatorname{Tr} S^{2} \phi^{2} \cdot \operatorname{Ric}-\operatorname{Tr} \phi S^{2} \operatorname{Ric} \cdot \phi .
\end{aligned}
$$

By the property (5.4) due to the Reeb invariant Ricci tensor $\mathcal{L}_{\xi}$ Ric $=0$, we have

$$
\phi S(\phi S \cdot \operatorname{Ric}-\mathrm{Ric} \cdot \phi S+\mathrm{Ric} \cdot S \phi-S \phi \mathrm{Ric})=0 .
$$

From this, by taking the trace, the first two terms become

$$
\operatorname{Tr}(\phi S)^{2} \cdot \operatorname{Ric}-\operatorname{Tr} \phi S \cdot \text { Ric } \cdot \phi S=\operatorname{Tr}(\phi S)^{2} \operatorname{Ric}-\operatorname{Tr}(\phi S)^{2} \operatorname{Ric}=0 .
$$

Then taking the trace of the next two terms gives

$$
\operatorname{Tr} \phi S \cdot \operatorname{Ric} \cdot S \phi=\operatorname{Tr} \phi S^{2} \phi \cdot \operatorname{Ric} .
$$

From the notion of Hopf, together with $(5.6)$ and (5.7), the equation $(5.5)$ can be changed as follows:

$$
\begin{aligned}
\operatorname{Tr}(\phi \cdot \operatorname{Ric}-\operatorname{Ric} \cdot \phi)^{2} & =-\operatorname{Tr}\left(\phi S^{2}-S^{2} \phi\right)(\phi \cdot \operatorname{Ric}-\operatorname{Ric} \cdot \phi) \\
& =\operatorname{Tr} \phi^{2} \cdot \operatorname{Ric} \cdot S^{2}+\operatorname{Tr} \phi^{2} S^{2} \cdot \operatorname{Ric}-2 \operatorname{Tr} \phi^{2} S \cdot \operatorname{Ric} \cdot S \\
& =2 \eta\left(\operatorname{Ric}\left(S^{2} \xi\right)\right)-2 \eta(S \cdot \operatorname{Ric}(S \xi)) \\
& =0,
\end{aligned}
$$

where we have used the equations

$$
\begin{aligned}
\operatorname{Tr} \phi^{2} \cdot \operatorname{Ric} \cdot S^{2} & =\operatorname{Tr}\left(-\operatorname{Ric} \cdot S^{2}+\eta\left(\operatorname{Ric} \cdot S^{2}\right) \xi\right) \\
& =-\operatorname{Tr} \operatorname{Ric} \cdot S^{2}+\eta\left(\operatorname{Ric}\left(S^{2} \xi\right)\right) \\
\operatorname{Tr} \phi^{2} \cdot S^{2} \cdot \operatorname{Ric} & =\operatorname{Tr}\left(-S^{2} \cdot \operatorname{Ric}+\eta\left(S^{2} \cdot \operatorname{Ric}\right) \xi\right) \\
& =-\operatorname{Tr} \operatorname{Ric} \cdot S^{2}+\eta\left(S^{2} \cdot \operatorname{Ric} \xi\right)
\end{aligned}
$$

and

$$
\begin{aligned}
-2 \operatorname{Tr} \phi^{2} S \cdot \operatorname{Ric} \cdot S & =-2 \operatorname{Tr}\left(-S \cdot \operatorname{Ric} \cdot S+\eta\left(S^{2} \cdot \operatorname{Ric}\right) \xi\right) \\
& =2 \operatorname{Tr} S \cdot \operatorname{Ric} \cdot S-2 \eta(S \cdot \operatorname{Ric}(S \xi)) .
\end{aligned}
$$

Moreover, by using our assumption of $N$ being $\mathfrak{A}$-isotropic, that is, $g(A N, N)=0$ and $g(A \xi, \xi)=0$, the third equality becomes

$$
\operatorname{Ric}(\xi)=\left\{-2(m-2)+h \alpha-\alpha^{2}\right\} \xi .
$$

From this we conclude that the Ricci tensor Ric commutes with the structure tensor $\phi$ in the case where the unit normal $N$ is $\mathfrak{A}$-isotropic. Then by Theorem $\mathrm{B}$ 
due to Suh and Hwang [36], we give a complete classification in our Main Theorem in the introduction.

\section{Proof of Main Theorem with a-Principal normal Vector Field}

In this section we want to prove our Main Theorem for real hypersurfaces in the complex hyperbolic quadric $Q^{m *}$ with commuting Ricci tensor and $\mathfrak{A}$-principal unit normal vector field. By the Ricci tensor given in the formula 4.1 for $\mathfrak{A}$-principal unit normal, that is, $A N=N$, we have

$$
\begin{aligned}
\operatorname{Ric}(\phi X)= & -(2 m-1) \phi X+A \phi X-g(A \phi X, N) A N \\
& +h S \phi X-S^{2} \phi X,
\end{aligned}
$$

and

$$
\begin{aligned}
\phi \operatorname{Ric}(X)= & -(2 m-1) \phi X+\phi A X-g(A X, N) \phi A N \\
& +h \phi S X-\phi S^{2} X,
\end{aligned}
$$

where the function $h$ denotes the trace of the shape operator $S$ of $M$ in $Q^{m *}$.

When we consider that the unit normal $N$ is $\mathfrak{A}$-principal, the unit normal $N$ is invariant under the complex conjugation $A$ in $\mathfrak{A}$, that is, $A N=N$ and $A \xi=-\xi$. By using such properties into (6.1) and $(6.2)$, we have

$$
\phi \cdot \operatorname{Ric}(X)-\operatorname{Ric} \cdot \phi(X)=\phi A X-A \phi X+h(\phi S-S \phi) X-\left(\phi S^{2}-S^{2} \phi\right) X .
$$

From this, together with $\mathcal{L}_{\xi}$ Ric $=0$, which is equivalent to $(\phi S-S \phi) \cdot \operatorname{Ric}=$ Ric $\cdot(\phi S-S \phi)$, we have

$$
\begin{aligned}
\operatorname{Tr}(\phi \cdot \operatorname{Ric}-\operatorname{Ric} \cdot \phi)^{2}= & h \operatorname{Tr}(\phi S-S \phi)(\phi \cdot \operatorname{Ric}-\text { Ric } \cdot \phi) \\
& -\operatorname{Tr}\left(\phi S^{2}-S^{2} \phi\right)(\phi \cdot \operatorname{Ric}-\text { Ric } \cdot \phi) \\
& +\operatorname{Tr}(\phi A-A \phi)(\phi \cdot \operatorname{Ric}-\operatorname{Ric} \cdot \phi) .
\end{aligned}
$$

On the other hand, since the complex conjugation is involutive and anti-commuting, such that $A J=-J A$, and the unit normal $N$ is $\mathfrak{A}$-invariant, it follows that

$$
\phi A=-A \phi .
$$

From this, together with $A \xi=-\xi$, we have

$$
\begin{aligned}
\operatorname{Tr} \phi A(\phi \cdot \operatorname{Ric}-\operatorname{Ric} \cdot \phi) & =-\operatorname{Tr} A \phi^{2} \cdot \operatorname{Ric}-\operatorname{Tr} \operatorname{Ric} \cdot \phi^{2} A \\
& =2 \operatorname{Tr} \operatorname{Ric} \cdot A-\eta(\operatorname{Ric}(A \xi))-\eta(A \cdot \operatorname{Ric}(\xi)) \\
& =2\{\operatorname{Tr} \operatorname{Ric} \cdot A+\eta(\operatorname{Ric}(\xi))\} .
\end{aligned}
$$

Then it follows that

$$
\begin{aligned}
\operatorname{Tr}(\phi \cdot \operatorname{Ric}-\operatorname{Ric} \cdot \phi)^{2}= & -\operatorname{Tr}\left(\phi S^{2}-S^{2} \phi\right)(\phi \cdot \operatorname{Ric}-\operatorname{Ric} \cdot \phi) \\
& +\operatorname{Tr}(\phi A-A \phi)(\phi \cdot \operatorname{Ric}-\operatorname{Ric} \cdot \phi) \\
= & 2 \eta\left(\operatorname{Ric} \cdot S^{2}(\xi)\right)-2 \eta(S \cdot \operatorname{Ric} \cdot S(\xi)) \\
& +4 \operatorname{Tr}(\operatorname{Ric} \cdot A)+4 \eta(\operatorname{Ric}(\xi)) .
\end{aligned}
$$


The Ricci tensor given in the formula 4.1 for $\mathfrak{A}$-principal unit normal, that is, $A N=N$ and $A \xi=-\xi$, gives

$$
\operatorname{Ric}(X)=-(2 m-1) X+2 \eta(X) \xi+A X+h S X-S^{2} X
$$

and

$$
\operatorname{Ric}(\xi)=\left\{-2(m-1)+h \alpha-\alpha^{2}\right\} \xi
$$

Then it follows that

$$
\operatorname{Ric}\left(e_{i}\right)=-(2 m-1) e_{i}+2 \eta\left(e_{i}\right) \xi+A e_{i}+h S e_{i}-S^{2} e_{i}
$$

and

$$
\operatorname{Ric}\left(A e_{i}\right)=-(2 m-1) e_{i}-2 \eta\left(e_{i}\right) \xi+e_{i}+h S A e_{i}-S^{2} A e_{i},
$$

where we have taken an orthonormal basis

$$
\left\{\xi, e_{1}, \ldots, e_{m-1}, \phi e_{1}, \ldots, \phi e_{m-1}\right\}
$$

of $T_{[z]} M,[z] \in M$, in $Q^{m *}$ such that $A e_{i}=e_{i}, A \phi e_{i}=-\phi e_{i}, A \xi=-\xi$, and $A N=N$. So it follows that

$$
\begin{aligned}
\operatorname{Tr}(\operatorname{Ric} \cdot A) & =g(A \xi, \operatorname{Ric}(\xi))+\sum_{i=1}^{2 m-2} g\left(A e_{i}, \operatorname{Ric}\left(e_{i}\right)\right) \\
& =-g(\xi, \operatorname{Ric}(\xi))+\sum_{i=1}^{m-1} g\left(A e_{i}, \operatorname{Ric}\left(e_{i}\right)\right)+\sum_{i=1}^{m-1} g\left(A \phi e_{i}, \operatorname{Ric}\left(\phi e_{i}\right)\right) .
\end{aligned}
$$

Substituting these ones into 6.3 and using the orthonormal basis, we have

$$
\begin{aligned}
\operatorname{Tr}(\phi \cdot \operatorname{Ric}-\operatorname{Ric} \cdot \phi)^{2} & =4 \sum_{i=1}^{m-1}\left\{g\left(\operatorname{Ric}\left(e_{i}\right), e_{i}\right)-g\left(\phi e_{i}, \operatorname{Ric}\left(\phi e_{i}\right)\right)\right\} \\
& =4\left\{\operatorname{Tr}^{*} \operatorname{Ric}+\operatorname{Tr}^{*} \phi \cdot \operatorname{Ric} \cdot \phi\right\} \\
& =4\left\{\operatorname{Tr}^{*} \operatorname{Ric}+\operatorname{Tr}^{*} \phi^{2} \cdot \operatorname{Ric}\right\} \\
& =4\left\{\operatorname{Tr}^{*} \operatorname{Ric}-\operatorname{Tr}^{*} \operatorname{Ric}\right\} \\
& =0
\end{aligned}
$$

where $\operatorname{Tr}^{*} \operatorname{Ric}=\sum_{i=1}^{m-1} g\left(\operatorname{Ric}\left(e_{i}\right), e_{i}\right)$ for the orthonormal basis $\left\{\xi, e_{1}, \ldots, e_{m-1}\right.$, $\left.\phi e_{1}, \ldots, \phi e_{m-1}\right\}$ of $T_{[z]} M,[z] \in M$, in $Q^{m *}$. Accordingly, we conclude that even for the $\mathfrak{A}$-principal normal the Ricci tensor Ric commutes with the structure tensor $\phi$, that is, Ric $\cdot \phi=\phi \cdot$ Ric. Then by Theorem B] due to Suh and Hwang [36, we give a complete classification of our main result.

\section{REFERENCES}

[1] J. Berndt, Real hypersurfaces with constant principal curvatures in complex hyperbolic space, J. Reine Angew. Math. 395 (1989), 132-141. MR 0983062

[2] J. Berndt, S. Console and C. E. Olmos, Submanifolds and holonomy, second edition, Monographs and Research Notes in Mathematics, CRC Press, Boca Raton, FL, 2016. MR 3468790.

[3] J. Berndt and Y. J. Suh, Contact hypersurfaces in Kähler manifolds, Proc. Amer. Math. Soc. 143 (2015), no. 6, 2637-2649. MR 3326043 
[4] J. Berndt, H. Lee and Y. J. Suh, Contact hypersurfaces in noncompact complex Grassmannians of rank two, Internat. J. Math. 24 (2013), no. 11, 1350089, 11 pp. MR 3143606

[5] A. L. Besse, Einstein manifolds, reprint of the 1987 edition, Classics in Mathematics, Springer-Verlag, Berlin, 2008. MR 2371700.

[6] T. E. Cecil and P. J. Ryan, Focal sets and real hypersurfaces in complex projective space, Trans. Amer. Math. Soc. 269 (1982), no. 2, 481-499. MR 0637703

[7] S. Helgason, Differential geometry, Lie groups, and symmetric spaces, corrected reprint of the 1978 original, Graduate Studies in Mathematics, 34, American Mathematical Society, Providence, RI, 2001. MR 1834454

[8] M. Kimura, Real hypersurfaces and complex submanifolds in complex projective space, Trans. Amer. Math. Soc. 296 (1986), no. 1, 137-149. MR 0837803

[9] M. Kimura and M. Ortega, Hopf real hypersurfaces in the indefinite complex projective space, Mediterr. J. Math. 16 (2019), no. 2, Paper No. 27, 16 pp. MR 3910061

[10] S. Klein, Totally geodesic submanifolds of the complex quadric, Differential Geom. Appl. 26 (2008), no. 1, 79-96. MR 2393975

[11] S. Klein and Y. J. Suh, Contact real hypersurfaces in the complex hyperbolic quadric, Ann. Mat. Pura Appl. (4) 198 (2019), no. 4, 1481-1494. MR 3987223

[12] A. W. Knapp, Lie groups beyond an introduction, second edition, Progress in Mathematics, 140, Birkhäuser Boston, Boston, MA, 2002. MR 1920389

[13] S. Kobayashi and K. Nomizu, Foundations of differential geometry. Vol. II, reprint of the 1969 original, Wiley Classics Library, John Wiley \& Sons, New York, 1996. MR 1393941.

[14] H. Lee and Y. J. Suh, Real hypersurfaces in the complex hyperbolic quadric with Reeb parallel structure Jacobi operator, Math. Phys. Anal. Geom. 23 (2020), no. 1, Paper No. 6, 18 pp. MR 4069855

[15] S. Montiel and A. Romero, Holomorphic sectional curvatures of indefinite complex Grassmann manifolds, Math. Proc. Cambridge Philos. Soc. 93 (1983), no. 1, 121-125. MR 0684280

[16] S. Montiel and A. Romero, Complex Einstein hypersurfaces of indefinite complex space forms, Math. Proc. Cambridge Philos. Soc. 94 (1983), no. 3, 495-508. MR 0720800

[17] S. Montiel and A. Romero, On some real hypersurfaces of a complex hyperbolic space, Geom. Dedicata 20 (1986), no. 2, 245-261. MR 0833849

[18] J. D. Pérez, Commutativity of Cho and structure Jacobi operators of a real hypersurface in a complex projective space, Ann. Mat. Pura Appl. (4) 194 (2015), no. 6, 1781-1794. MR 3419917

[19] J. D. Pérez, On the Riemannian curvature tensor of a real hypersurface in a complex projective space, Math. Nachr. 289 (2016), no. 17-18, 2263-2272. MR 3583268.

[20] J. D. Pérez, Lie derivatives and structure Jacobi operator on real hypersurfaces in complex projective spaces, Differential Geom. Appl. 50 (2017), 1-10. MR 3588636

[21] J. D. Pérez and Y. J. Suh, The Ricci tensor of real hypersurfaces in complex two-plane Grassmannians, J. Korean Math. Soc. 44 (2007), no. 1, 211-235. MR 2283469

[22] J. D. Pérez, Y. J. Suh and Y. Watanabe, Generalized Einstein real hypersurfaces in complex two-plane Grassmannians, J. Geom. Phys. 60 (2010), no. 11, 1806-1818. MR 2679423

[23] H. Reckziegel, On the geometry of the complex quadric, in Geometry and topology of submanifolds, VIII (Brussels, 1995/Nordfjordeid, 1995), 302-315, World Sci. Publ., River Edge, NJ, 1996. MR 1434581

[24] A. Romero, On a certain class of complex Einstein hypersurfaces in indefinite complex space forms, Math. Z. 192 (1986), no. 4, 627-635. MR 0847011 
[25] B. Smyth, Differential geometry of complex hypersurfaces, Ann. of Math. (2) 85 (1967), 246-266. MR 0206881

[26] B. Smyth, Homogeneous complex hypersurfaces, J. Math. Soc. Japan 20 (1968), 643-647. MR 0232326

[27] Y. J. Suh, Real hypersurfaces of type $B$ in complex two-plane Grassmannians, Monatsh. Math. 147 (2006), no. 4, 337-355. MR 2215841

[28] Y. J. Suh, Real hypersurfaces in complex two-plane Grassmannians with commuting Ricci tensor, J. Geom. Phys. 60 (2010), no. 11, 1792-1805. MR 2679422

[29] Y. J. Suh, Real hypersurfaces in complex two-plane Grassmannians with parallel Ricci tensor, Proc. Roy. Soc. Edinburgh Sect. A 142 (2012), no. 6, 1309-1324. MR 3002598

[30] Y. J. Suh, Hypersurfaces with isometric Reeb flow in complex hyperbolic two-plane Grassmannians, Adv. in Appl. Math. 50 (2013), no. 4, 645-659. MR 3032310

[31] Y. J. Suh, Real hypersurfaces in the complex quadric with Reeb parallel shape operator, Internat. J. Math. 25 (2014), no. 6, 1450059, 17 pp. MR 3225583

[32] Y. J. Suh, Real hypersurfaces in the complex hyperbolic quadric with parallel normal Jacobi operator, Mediterr. J. Math. 15 (2018), no. 4, Paper No. 159, 14 pp. MR 3816192

[33] Y. J. Suh, Real hypersurfaces in the complex hyperbolic quadrics with isometric Reeb flow, Commun. Contemp. Math. 20 (2018), no. 2, 1750031, 20 pp. MR 3730753

[34] Y. J. Suh and D. H. Hwang, Real hypersurfaces in the complex hyperbolic quadric with Reeb parallel shape operator, Ann. Mat. Pura Appl. (4) 196 (2017), no. 4, 1307-1326. MR 3673668

[35] Y. J. Suh and D. H. Hwang, Real hypersurfaces in the complex quadric with commuting Ricci tensor, Sci. China Math. 59 (2016), no. 11, 2185-2198. MR 3561833

[36] Y. J. Suh and D. H. Hwang, Real hypersurfaces in the complex hyperbolic quadric with commuting Ricci tensor, Bull. Malays. Math. Sci. Soc. 42 (2019), no. 3, 1173-1198. MR 3942454

\section{Doo Hyun Hwang}

The Research Institute of Real and Complex Manifolds (RIRCM), Kyungpook National University, Daegu 41566, Republic of Korea

engus0322@knu.ac.kr

Hyunjin Lee ${ }^{凶}$

The Research Institute of Real and Complex Manifolds (RIRCM), Kyungpook National University, Daegu 41566, Republic of Korea

lhjibis@hanmail.net

Young Jin Suh

Department of Mathematics \& RIRCM, Kyungpook National University, Daegu 41566, Republic of Korea

yjsuh@knu.ac.kr

Received: April 10, 2020

Accepted: June 25, 2020 